\title{
Effects of hypothermia on inflammatory cytokine expression in rat liver following asphyxial cardiac arrest
}

\author{
YOONSOO PARK $^{1 *}$, JI HYEON AHN ${ }^{2,3 *}$, JEONG HWI CHO $^{4}$, HYUN-JIN TAE $^{4}$, TAE-KYEONG LEE ${ }^{5}$, \\ BORA KIM ${ }^{3}$, JAE-CHUL LEE ${ }^{3}$, JOON HA PARK ${ }^{6}$, MYOUNG CHEOL SHIN ${ }^{1}$, \\ TAEK GEUN OHK ${ }^{1}$, JUN HWI CHO ${ }^{1}$ and MOO-HO WON ${ }^{3}$
}

\begin{abstract}
${ }^{1}$ Department of Emergency Medicine, Kangwon National University Hospital, School of Medicine, Kangwon National University, Chuncheon, Gangwon 24289; ${ }^{2}$ Department of Physical Therapy, College of Health Science, Youngsan University, Yangsan, Gyeongnam 50510; ${ }^{3}$ Department of Neurobiology, School of Medicine, Kangwon National University, Chuncheon, Gangwon 24341; ${ }^{4}$ Bio-Safety Research Institute, College of Veterinary Medicine, Chonbuk National University, Iksan, Jeollabuk 54596; ${ }^{5}$ Department of Biomedical Science and Research Institute for Bioscience and Biotechnology, Hallym University, Chuncheon, Gangwon 24252; ${ }^{6}$ Department of Anatomy, College of Korean Medicine, Dongguk University, Gyeongju, Gyeongbuk 38066, Republic of Korea
\end{abstract}

Received August 6, 2020; Accepted March 17, 2021

DOI: $10.3892 / \mathrm{etm} .2021 .10058$

\begin{abstract}
Hypothermic treatment is known to protect against cardiac arrest (CA) and improve survival rate. However, few studies have evaluated the CA-induced liver damage and the effects of hypothermia on this damage. Therefore, the aim of the present study was to determine possible protective effects of hypothermia on the liver after asphyxial CA. Rats were subjected to a 5-min asphyxial CA followed by return of spontaneous circulation (ROSC). The body temperature was controlled at $37 \pm 0.5^{\circ} \mathrm{C}$ (normothermia group) or $33 \pm 0.5^{\circ} \mathrm{C}$ (hypothermia group) for $4 \mathrm{~h}$ after ROSC. Livers were examined at 6,12 h, 1 and 2 days after ROSC. Histopathological examination was performed by H\&E staining. Alterations in the expression levels of pro-inflammatory (TNF- $\alpha$ and interleukin IL-2) and anti-inflammatory cytokines (IL-4 and IL-13) were investigated by immunohistochemistry. Sinusoidal dilatation and vacuolization were observed after asphyxial CA
\end{abstract}

Correspondence to: Professor Moo-Ho Won, Department of Neurobiology, School of Medicine, Kangwon National University, 1 Kangwondaehak-gil, Chuncheon, Gangwon 24341, Republic of Korea

E-mail:mhwon@kangwon.ac.kr

Professor Jun Hwi Cho, Department of Emergency Medicine, Kangwon National University Hospital, School of Medicine, Kangwon National University, 156 Baengnyeong-ro, Chuncheon, Gangwon 24389, Republic of Korea

E-mail: cjhemd@kangwon.ac.kr

*Contributed equally

Key words: anti-inflammatory cytokines, asphyxial cardiac arrest, hypothermia, liver, pro-inflammatory cytokines by histopathological examination. However, these CA-induced structural alterations were prevented by hypothermia. In immunohistochemical examination, the expression levels of pro-inflammatory cytokines were reduced in the hypothermia group compared with those in the normothermia group while the expression levels of anti-inflammatory cytokines were increased in the hypothermia group compared with those in the normothermia group. In conclusion, hypothermic treatment for $4 \mathrm{~h}$ following asphyxial $\mathrm{CA}$ in rats inhibited the increase of pro-inflammatory cytokines and stimulated the expression of anti-inflammatory cytokines compared with the normothermic group. The results of the present study suggested that hypothermic treatment after asphyxial CA reduced liver damage via the regulation of inflammation.

\section{Introduction}

Liver ischemia-reperfusion (IR) injury occurs in diverse clinical settings, including liver hemorrhage and shock, surgical resection, transplantation and CA $(1,2)$. Among these diverse clinical settings, cardiac arrest (CA) is known to result in high rates of mortality (ranging from 46 to 68\%) (3). Therapeutic hypothermia can improve the survival rate and the neurological outcomes of post-CA patients for several years $(4,5)$. Hypothermia can markedly reduce ischemia-associated tissue damage in various organs, such as the liver, kidney and heart $(6,7)$. Among these organs, the liver requires mitochondrial oxidative phosphorylation for its energy supply; therefore, the liver is a target of IR injury (8).

During the IR process, hepatocyte injury occurs through several pathways, such as lipid peroxidation, the release of reactive oxygen species (ROS), the activation of signal transduction cascades and the production of diverse inflammatory mediators (9). An IR event to an organ causes an acute inflammatory response that results in important cellular damage and organ dysfunction (10). At an early stage after injury, 
the production of pro-inflammatory cytokines is known to be increased $(11,12)$. Inflammatory responses in the liver IR injury involve complex interactions between various cellular and humoral contributors (7). Kupffer cells, neutrophils and hepatocytes are major cellular players (8). Pro-inflammatory and anti-inflammatory cytokines, chemokines and complement proteins are also involved in the IR-induced liver injury (8). Therefore, it is fundamental to elucidate the inflammatory process for preventing the liver IR injury following CA.

The beneficial effects of hypothermia include the decreased hepatic metabolism, the subsequent reduction in oxygen requirement and the inflammatory response regulation (13-15). Pretreatment with mild hypothermia before ischemia can reduce apoptosis and the production of inflammatory cytokines. Posttreatment with hypothermia after ischemic events is sufficient to reduce IR injury by inhibiting inflammatory responses (16). It has been reported that mild hypothermic treatment $\left(35^{\circ} \mathrm{C}\right)$ for $1-24 \mathrm{~h}$ after $60 \mathrm{~min}$ of ischemia enzymatically improved liver function in a rat model of liver ischemia (17). In addition, hypothermia or spontaneous cooling for several to $24 \mathrm{~h}$ beginning immediately after CA could improve liver function and reduce death in rats (17).

To date, it has been reported that hypothermic therapy can effectively reduce the histopathological damage of lumbar spinal cord $(18,19)$, hippocampus $(20)$ and cerebellum (21) in the brain of a rat model of asphyxial CA. However, as aforementioned, few studies have examined the effects of hypothermia on the liver after CA. In addition, the therapeutic or protective mechanisms of hypothermic treatment in the liver after CA are still not well understood. Therefore, the objective of the present study was to chronically observe the tissue damage and the alterations in the expression levels of pro-inflammatory (TNF- $\alpha$ and IL-2) and anti-inflammatory cytokines (IL-4 and IL-13) in the liver following $5 \mathrm{~min}$ of asphyxial CA in rats. The effects of hypothermic treatment on tissue damage and the expression levels of pro- and anti-inflammatory cytokines in the liver after CA were also investigated.

\section{Materials and methods}

Experimental animals and groups. Male Sprague-Dawley rats (age, 10 weeks; body weight, 310-320 g) were obtained from the Experimental Animal Center of Kangwon National University (Chuncheon, South Korea). The rats were maintained in pathogen-free conditions under appropriate temperature $\left(\sim 23^{\circ} \mathrm{C}\right)$, humidity $(\sim 60 \%)$ and 12 -h light/dark cycle with freely accessible pellet feed and water. All experimental protocols used in the present study were approved on the basis of ethical procedures and scientific care proposed by the Institutional Animal Care and Use Committee of Kangwon National University (approval no. KW-180124-1; January 2018; Chuncheon, South Korea). Proper animal handling and care conformed to the guidelines of the current international laws and policies [NIH Guide for the Care and Use of Laboratory Animals, The National Academies Press, 8th Edition, 2011 (22); AVMA Guidelines for the Euthanasia of Animals: 2013 Edition (23)]. The number of rats used in the current study and the suffering caused by the procedures performed in all experiments were minimized. The body weight and the behavior of all animals were monitored every other day. Humane endpoints were determined when the animals exhibited $>20 \%$ weight loss, dehydration and loss of ability to ambulate. No animals presented signs of humane endpoints intended to be euthanized immediately. Euthanasia involved chemical and physical methods. Cardiac perfusion was conducted after each animal was profoundly anesthetized using $60 \mathrm{mg} / \mathrm{kg}$ pentobarbital sodium. Death was confirmed by evaluating vital signs, including heartbeat, pupillary response and respiratory pattern (lack of cardiac activity for 5 min through cardiac palpation, unresponsiveness to light with dilated pupils after directing light into the eyes of the animal and lack of a spontaneous breathing pattern with a shallow and irregular breathing pattern).

The rats $(n=101)$ used in the current study were randomly divided into the following groups: i) Normal group ( $n=5$; data not shown); ii) sham-operated normothermia group ( $\mathrm{n}=5$ at each time point), which was not subjected to CA operation, and the body temperature was controlled at $37 \pm 0.5^{\circ} \mathrm{C}$ for $4 \mathrm{~h}$; iii) CA-operated normothermia group ( $n=7$ at each time point), which was subjected to $\mathrm{CA}$, and the body temperature was controlled at $37 \pm 0.5^{\circ} \mathrm{C}$ for $4 \mathrm{~h}$ after ROSC; iv) sham-operated hypothermia group ( $\mathrm{n}=5$ at each time point), which was not subjected to $\mathrm{CA}$ operation, and the body temperature was controlled at $33.0 \pm 0.5^{\circ} \mathrm{C}$ for $4 \mathrm{~h}$; and v) CA-operated hypothermia group ( $n=7$ at each time point), which was subjected to $\mathrm{CA}$, and the body temperature was controlled at $33.0 \pm 0.5^{\circ} \mathrm{C}$ for $4 \mathrm{~h}$ after ROSC. The rats in each group were sacrificed at 6, 12 h, 1 and 2 days after ROSC.

$C A$ induction and cardiopulmonary resuscitation (CPR). CA and CPR were performed according to previously published protocols $(24,25)$ with minor modifications. Briefly, the rats were anesthetized with $2-3 \%$ isoflurane and mechanically ventilated to maintain respiration using a rodent ventilator (Harvard Apparatus). Body temperature was maintained at $37 \pm 0.5^{\circ} \mathrm{C}$ during $\mathrm{CA}$ surgery. Peripheral oxygen saturation was monitored with an oxygen saturation probe of pulse oximetry (Nonin Medical Inc.), which was attached to the left foot. To record the electrocardiogram, electrocardiographic probes (GE Healthcare) were placed in the four limbs, and the data were monitored continuously. Simultaneously, the left femoral artery and right femoral vein were separately cannulated to monitor the mean arterial pressure (MAP) and intravenous injections (MLT 1050/D; ADInstruments Ltd.).

A total of $2 \mathrm{mg} / \mathrm{kg}$ vecuronium bromide (Gensia Sicor Pharmaceuticals, Inc.) was intravenously administered after a 5-min stabilization period, anesthesia was stopped, and mechanical ventilation was halted. At this point, MAP was $<25 \mathrm{mmHg}$, and subsequent pulseless electric activity and asystolic rhythm were used to define CA (26). CA was confirmed at 3-4 min after the injection of vecuronium bromide and was performed for $5 \mathrm{~min}$. CPR was initiated by intravenously administering $0.005 \mathrm{mg} / \mathrm{kg}$ epinephrine and $1 \mathrm{mEq} / \mathrm{kg}$ sodium bicarbonate. Mechanical ventilation with $100 \%$ oxygen and mechanical chest compression were provided at a rate of 300/min until MAP reached $60 \mathrm{~mm} \mathrm{Hg}$ and electrocardiographic activity was observed. If ROSC was not detected, half the amount of epinephrine was administered during a 1-min CPR; however, the rats subjected to a third CPR were excluded from the present study. In the normothermic 
groups, body temperature was maintained at $37 \pm 0.5^{\circ} \mathrm{C}$ after the CA surgery.

Hypothermic treatment. According to published protocols $(21,27)$, hypothermic treatment was performed after ROSC. In brief, hypothermic treatment was provided by cooling the body surface with isopropyl alcohol wipes, ice packs, an electrical fan and a cooling blanket. Body temperature in the hypothermia groups was maintained at $33 \pm 0.5^{\circ} \mathrm{C}$ for $4 \mathrm{~h}$ by rectal temperature sensor monitoring. The rats were re-warmed to $37 \pm 0.5^{\circ} \mathrm{C}$ for 30 min using a warming blanket and a hot pad.

Tissue preparation. According to our previous study (28), the rats were anesthetized by intraperitoneal administration of sodium pentobarbital $(60 \mathrm{mg} / \mathrm{kg}$; JW Pharmaceutical Corp.) (29), and the vital signs of the animals were examined to ensure that the animals were profoundly anesthetized. After anesthesia, the rats were transcardially perfused with $0.1 \mathrm{M}$ PBS ( $\mathrm{pH} 7.4$ ), followed by perfusion with $4 \%$ paraformaldehyde solution (diluted in 0.1 M PBS, pH 7.4). Their livers were isolated and post-fixed with the same fixative for $24 \mathrm{~h}$ at room temperature. Subsequently, the liver tissues were cut, embedded in paraffin and sectioned into $6-\mu \mathrm{m}$ thickness. Finally, the sections were mounted on gelatin-coated microscopy slides.

$H \&$ E staining. CA-induced pathological alterations in the livers of each group were examined by H\&E staining according to our previous study (28). In brief, the paraffin sections were deparaffinized in xylene and rehydrated in a descending ethanol gradient and washed briefly in distilled water. Next, the sections were stained with hematoxylin solution for $7 \mathrm{~min}$ followed by eosin solution for $5 \mathrm{~min}$ at room temperature and dehydrated by immersion in a descending ethanol gradient. Finally, they were mounted with Canada balsam (Kanto Chemical Co., Inc.).

CA-induced damage and the hypothermic effect on the CA-induced damage were analyzed under AxioM1 light microscope (magnification, x20; Carl Zeiss AG,) equipped with a digital camera (Axiocam; Carl Zeiss AG) connected to a PC monitor.

Immunohistochemistry. Immunohistochemistry was carried out to examine alterations in the expression of proinflammatory (TNF- $\alpha$ and IL-2) and anti-inflammatory cytokines (IL-4 and IL-13). According to our previous study (30), the paraffin sections were deparaffinized in xylene and rehydrated using a descending ethanol gradient and washed briefly in distilled water. Thereafter, endogenous peroxidase activity was blocked with $0.3 \%$ hydrogen peroxide $\left(\mathrm{H}_{2} \mathrm{O}_{2}\right)$ for $20 \mathrm{~min}$ at room temperature. Antigen retrieval was performed for 5 min at $95^{\circ} \mathrm{C}$ in sodium citrate buffer. Unspecific proteins were blocked using 5\% normal donkey serum (Vector Laboratories Inc.) for $30 \mathrm{~min}$ at room temperature. Next, the sections were incubated with solutions of the following primary antibodies overnight at $4{ }^{\circ} \mathrm{C}$ : Rabbit anti-TNF- $\alpha$ (Cat. no. ab66579, diluted 1:500; Abcam), mouse anti-IL-2 (Cat. no. sc-133118, diluted 1:200; Santa Cruz Biotechnology, Inc.), mouse anti-IL-4 (Cat. no. sc-53084, diluted 1:200;
Santa Cruz Biotechnology, Inc.) and mouse anti-IL-13 (Cat. no. sc-393365, diluted 1:200; Santa Cruz Biotechnology, Inc.). The sections were subsequently incubated with the secondary antibody solution (1:250, Vector Laboratories Inc.), biotinylated goat-anti rabbit (Cat. no. BA-1000) or horse anti-mouse IgG (Cat. no. BA-2000) for $60 \mathrm{~min}$ at room temperature and developed using VECTASTAIN ABC kit, which contains the avidin/biotin-based peroxidase system (Vector Laboratories Inc.). Finally, the signal was visualized with 3,3'-diaminobenzidine, and the sections were dehydrated and mounted with Canada balsam.

Quantitative analyses of TNF- $\alpha$, IL-2, IL-4 and IL-13 immunoreactivity in each group were performed according to our previous study (28). Briefly, images of TNF- $\alpha$, IL-2, IL-4 and IL-13-immunoreactive structures were captured using AxioM1 light microscope (magnification, 20x) equipped with a digital camera connected to a PC monitor. The density of each immunoreactive structure in the liver tissue section was evaluated as relative optical density (ROD) using ImageJ v1.59 software (National Institutes of Health). The results are presented as a ratio of ROD (\%) vs. the sham-operated group.

Statistical analysis. All statistical analyses were performed with GraphPad InStat (v3.05; GraphPad Software, Inc.) and data are presented as the mean \pm SD for physiological variables and mean \pm SEM for ROD. Differences between the groups were assessed using one-way ANOVA followed by Tukey's post hoc test. $\mathrm{P}<0.05$ was considered to indicate a statistically significant difference.

\section{Results}

Physiological variables and survival rate. Before CA operation, all the animals in the CA-operated normothermia and hypothermia groups exhibited normal physiological values of body weight, temperature, heart rate and MAP (Table I). In addition, at 2 days after ROSC, the survival rate was $14.3 \%$ in the CA-operated normothermia group (the survival rate immediately after ROSC was $85.7 \%$ ), while it was $42.9 \%$ in the CA-operated hypothermia groups (the survival rate immediately after ROSC was 100\%).

$H \& E$ staining. Normal rat liver histology was observed in the sham-operated normothermia and hypothermia groups (Fig. 1A and a). In the CA-operated normothermia group, hepatocyte swelling was detected at $6 \mathrm{~h}$ after CA (Fig. 1B). From $12 \mathrm{~h}$ after CA, sinusoidal dilatation, vacuolization and infiltration of inflammatory cells in the interstitial tissue were observed (Fig. 1C-E). On the other hand, in the CA-operated hypothermia group, structural alterations, such as those in the CA-operated normothermia group, were less profound compared with those in the CA-operated normothermia group (Fig. 1b-e).

TNF- $\alpha$ immunoreactivity. In the sham-operated normothermia and hypothermia groups, weak TNF- $\alpha$ immunoreactivity was detected in the rat liver (Fig. 2A and a), and this finding did not differ from that in the normal group (data not shown).

In the CA-operated normothermia group, the level of TNF- $\alpha$ immunoreactivity progressively increased in 
Table I. Physiological variables in the CA-operated normothermia and hypothermia groups.

\begin{tabular}{lcccc}
\hline & Body weight $(\mathrm{g})$ & Temperature $\left({ }^{\circ} \mathrm{C}\right)$ & Heart rate $($ beats $/ \mathrm{min})$ & $\mathrm{MAP}(\mathrm{mmHg})$ \\
\hline $\mathrm{CA}+$ normothermia & $303.3 \pm 3.9$ & $36.4 \pm 0.2$ & $348.8 \pm 4.2$ & $117.0 \pm 1.1$ \\
$\mathrm{CA}+$ hypothermia & $301.6 \pm 1.4$ & $36.5 \pm 0.1$ & $346.5 \pm 1.7$ & $114.2 \pm 1.9$ \\
\hline
\end{tabular}

Data are presented as the mean $\pm \mathrm{SD}$ for each group. CA, cardiac arrest; CPR, cardiopulmonary resuscitation; MAP, mean arterial pressure.
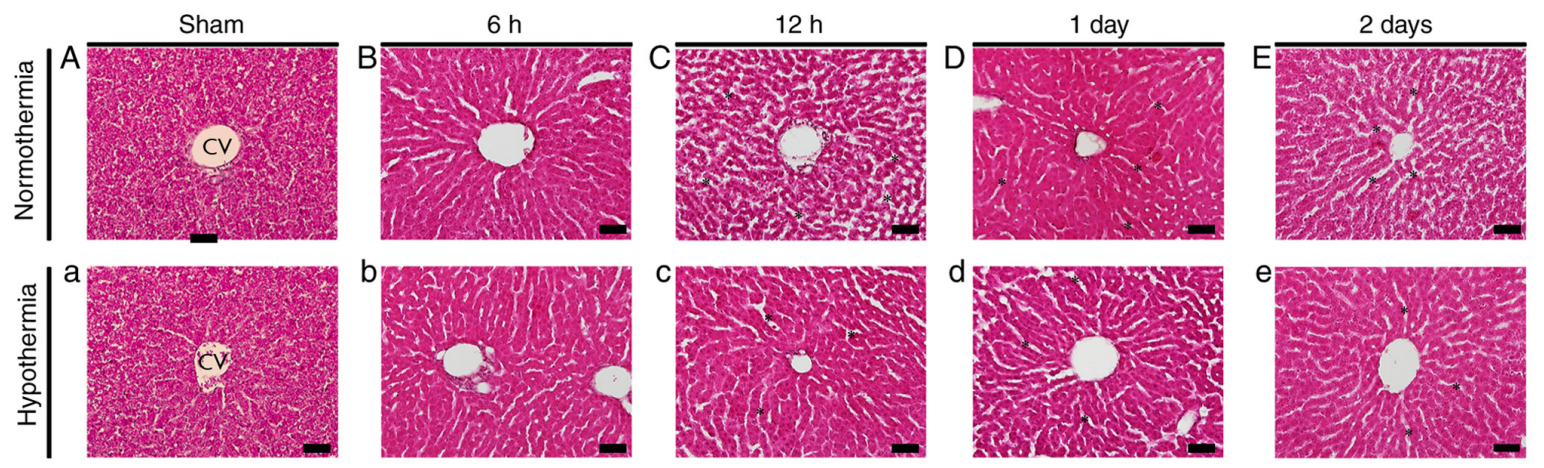

Figure 1. H\&E staining in the liver of the normothermia and hypothermia groups. (A-E) In histopathological examination, there were structural alterations, including hepatocyte swelling and sinusoidal dilatation (asterisk) from $6 \mathrm{~h}$ after CA in the normothermia group, (a-e) whereas these alterations were decreased in the hypothermia group. Scale bar, $50 \mu \mathrm{m}$. CV, central vein.
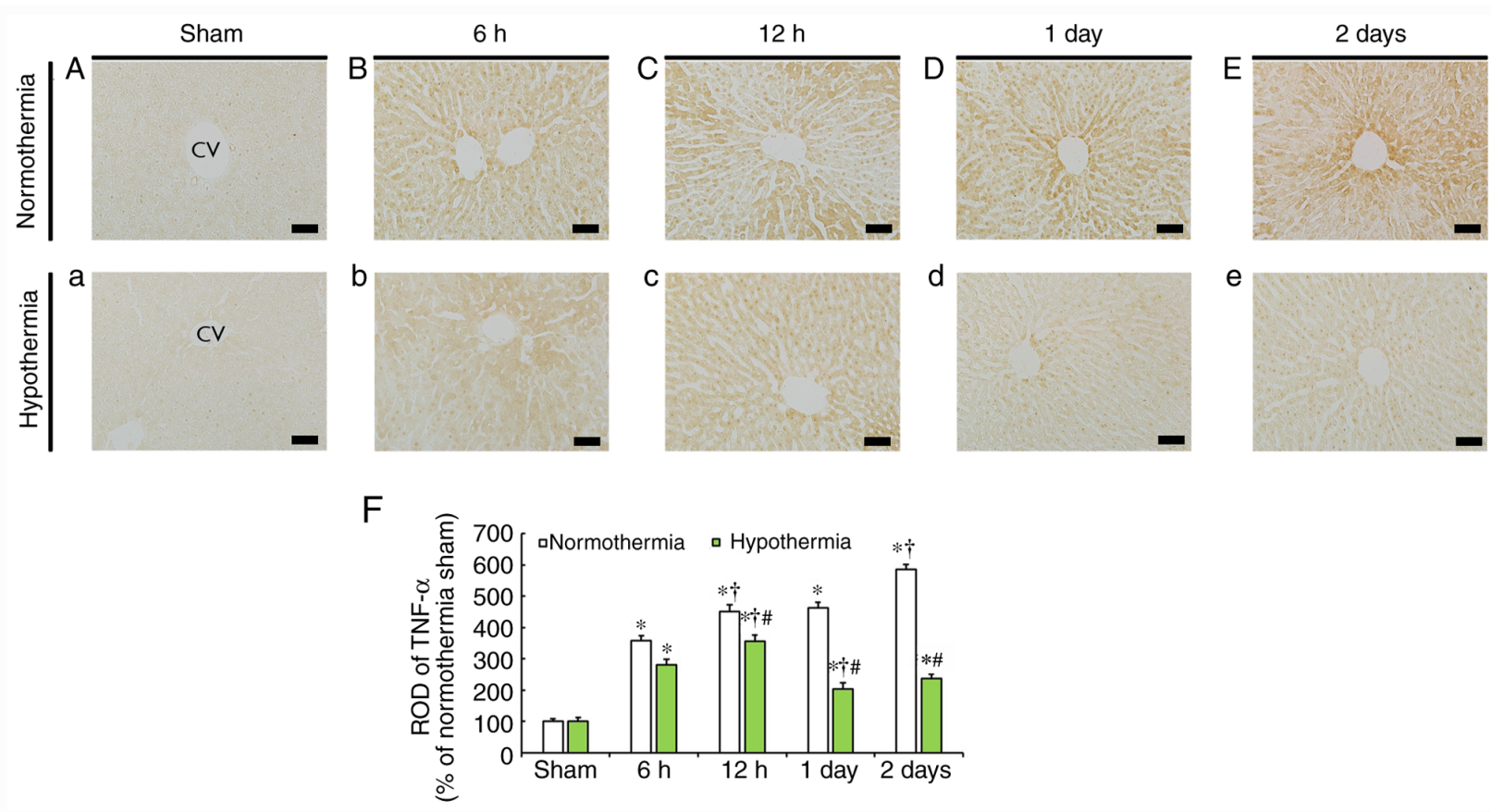

Figure 2. TNF- $\alpha$ immunoreactivity in the liver of the normothermia and hypothermia groups. (A-E) In the CA-operated normothermia group, TNF- $\alpha$ immunoreactivity was progressively increased in hepatocytes from $6 \mathrm{~h}$ after CA; (a-e) however, TNF- $\alpha$ immunoreactivity in the CA-operated hypothermia group was lower compared with that at the corresponding time points of the CA-operated normothermia group. Scale bar, $50 \mu \mathrm{m}$. (F) ROD of TNF- $\alpha$ immunoreactivity in the liver of the normothermia and hypothermia groups. $\mathrm{n}=5$ (sham) or 7 (CA) per group. " $\mathrm{P}<0.05$ vs. the sham-operated normothermia or hypothermia group; ${ }^{\dagger} \mathrm{P}<0.05$ vs. the same group at the previous time point; ${ }^{*} \mathrm{P}<0.05$ vs. the normothermia group at the same time point. The data are presented as the mean \pm SEM. $\mathrm{CV}$, central vein; ROD, relative optical density.

hepatocytes from $6 \mathrm{~h}$ to 2 days after $\mathrm{CA}$ in a time-dependent manner (Fig. 2B-E), and the mean ROD of TNF- $\alpha$ immunoreactivity was indicated to be $358.9,450.4,462.9$ and $586.2 \%$ at
6, $12 \mathrm{~h}, 1$ and 2 days post-CA, respectively, compared with that in the sham-operated normothermia group (Fig. 2F).

TNF- $\alpha$ immunoreactivity in the CA-operated hypothermia group also increased from 6 to $12 \mathrm{~h}$ after CA (Fig. $2 \mathrm{~b}$ and c), but 

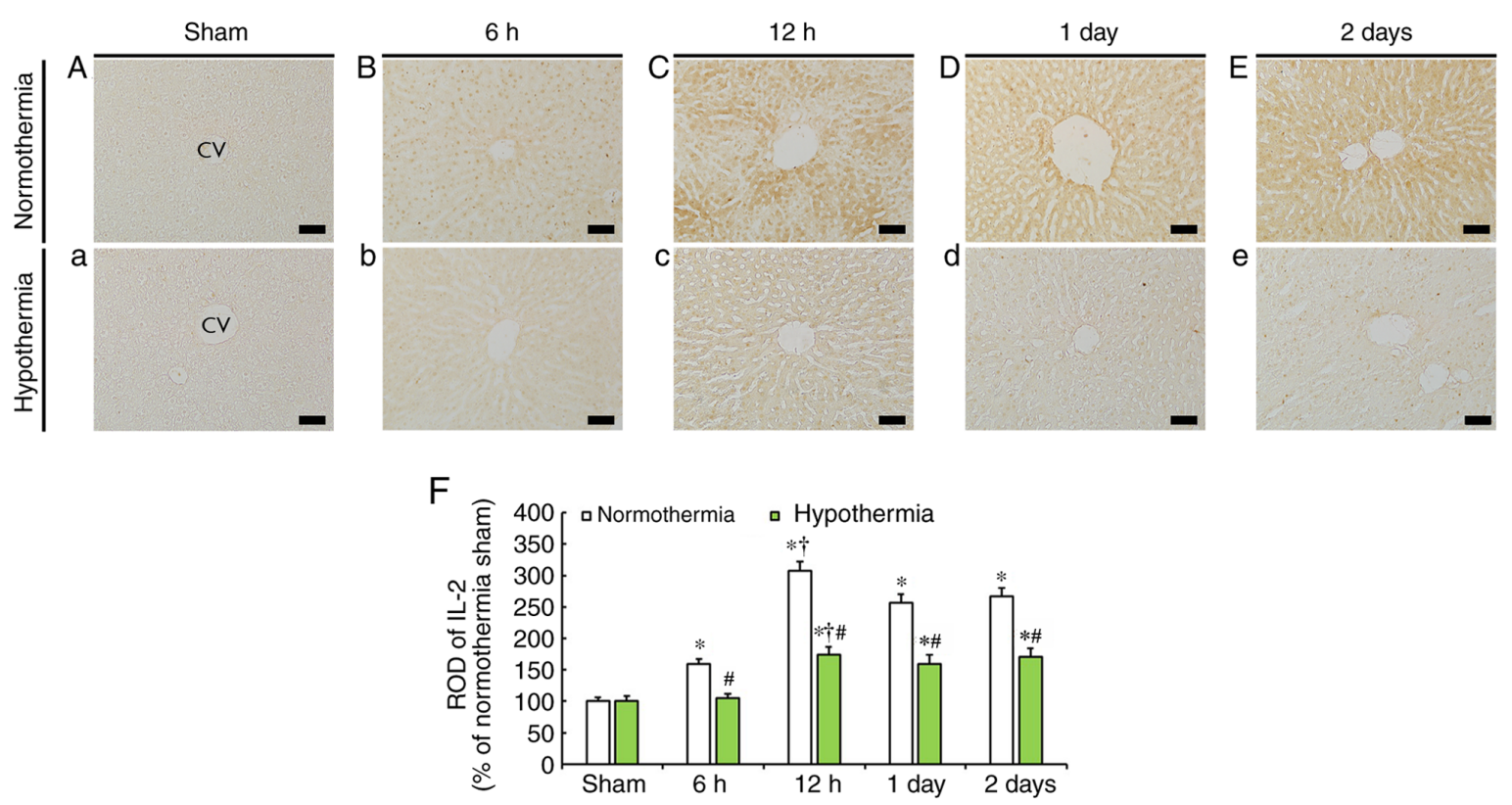

Figure 3. Immunoreactivity of IL-2 in the liver tissue of the normothermia group and hypothermia groups. (A-E) In the CA-operated normothermia group, IL-2 immunoreactivity was increased from $6 \mathrm{~h}$ post-CA and reached its highest level at $12 \mathrm{~h}$ post-CA, while the immunoreactivity was reduced in the subsequent time points. (a-e) In the CA-operated hypothermia group, IL-2 immunoreactivity was lower than that at the corresponding time points of the CA-operated normothermia group. Scale bar, $50 \mu \mathrm{m}$. (F) ROD of IL-2 immunoreactivity in the normothermia and hypothermia groups. $\mathrm{n}=5$ (sham) or 7 (CA) per group. ${ }^{*} \mathrm{P}<0.05$ vs. the sham-operated normothermia or hypothermia group; ${ }^{\dagger} \mathrm{P}<0.05$ vs. the same group at the previous time point; ${ }^{\sharp} \mathrm{P}<0.05$ vs. the normothermia group at the same time point. The data are presented as the mean \pm SEM. CV, central vein; ROD, relative optical density.

ROD was significantly lower (280.7 and $355.2 \%$, respectively) compared with that at the corresponding time points of the CA-operatednormothermiagroup(Fig.2F).Subsequently,TNF- $\alpha$ immunoreactivity was markedly decreased (Fig. 2d and e), with ROD being $203.7 \%$ at 1 day and $248.2 \%$ at 2 days, respectively, compared with that at corresponding time of the CA-operated normothermia groups (Fig. 2F).

IL-2 immunoreactivity. Weak IL-2 immunoreactivity was observed in the liver of the sham-operated normothermia and hypothermia groups (Fig. 3A and a).

IL-2 immunoreactivity in the liver of the CA-operated normothermia group was significantly increased at $6 \mathrm{~h}$ (159.7\% of the sham-operated normothermia group) and reach the highest value at $12 \mathrm{~h}(307.3 \%$ of the sham-operated normothermia group) after CA (Fig. 3B, C and F). At the following time points, the increased IL-2 immunoreactivity was reduced, but was still higher than that in the sham-operated normothermia group (Fig. 3D and E), with ROD being 256.9 and $267.1 \%$ of the sham-operated normothermia group, respectively, at 1 and 2 days post-CA (Fig. 3F).

In the CA-operated hypothermia group, IL-2 immunoreactivity at $6 \mathrm{~h}$ post-CA was similar to that in the sham-operated hypothermia group (Fig. 3b). In the subsequent time points, IL-2 immunoreactivity was increased compared with that of the sham-operated hypothermia group (Fig. 3c-e); however, ROD at 12 h, 1 and 2 days post-CA was significantly lower (174.2, 159.6 and $170.9 \%$, respectively) compared with that at the corresponding time points of the CA-operated normothermia group (Fig. 3F).

IL-4 immunoreactivity. IL-4 immunoreactivity was generally evident in the liver, and the level of immunoreactivity was mainly observed around the central veins in both sham-operated normothermia and hypothermia groups (Fig. 4A and a).

In the CA-operated normothermia group, IL-4 immunoreactivity gradually increased from $6 \mathrm{~h}$ after $\mathrm{CA}$ and reached the highest level at 1 day after CA (Fig. 4B-D), with ROD at 6, $12 \mathrm{~h}$ and 1 day post-CA being 148.1, 239.0 and 296.6\% of that in the sham-operated normothermia group (Fig. 4F). At 2 days after CA, IL-4 immunoreactivity in the liver was notably decreased (Fig. 4E and F; $85.6 \%$ of that in the sham-operated normothermia group).

In the CA-operated hypothermia group, the pattern of IL-4 immunoreactivity was similar to that in the CA-operated normothermia group (Fig. 4b-e); however, IL-4 immunoreactivity in this group was higher by $12.7 \%$ at $6 \mathrm{~h}, 56.8 \%$ at $12 \mathrm{~h}, 33.8 \%$ at 1 day and $16.4 \%$ at 2 days post-CA compared with that at the corresponding time points of the CA-operated normothermia group (Fig. 4F).

IL-13 immunoreactivity. IL-13 immunoreactivity was evident in the liver of the sham-operated normothermia and hypothermia groups and was mainly observed around the central veins (Fig. 5A and a).

IL-13 immunoreactivity in the CA-operated normothermia group was slightly decreased at $6 \mathrm{~h}$ post-CA (Fig. 5B) and recovered at $12 \mathrm{~h}$ post-CA (Fig. $5 \mathrm{C}$ and $\mathrm{F}$ ). In the subsequent time points, IL-13 immunoreactivity was significantly increased by $244.8 \%$ at 1 day and by $37.5 \%$ at 2 days post-CA (Fig. 5D-F) compared with that in the sham-operated normothermia group.

In the CA-operated hypothermia group, IL-13 immunoreactivity was slightly increased at $6 \mathrm{~h}$ post-CA $(127.9 \%$ of the sham-operated hypothermia group) and significantly 

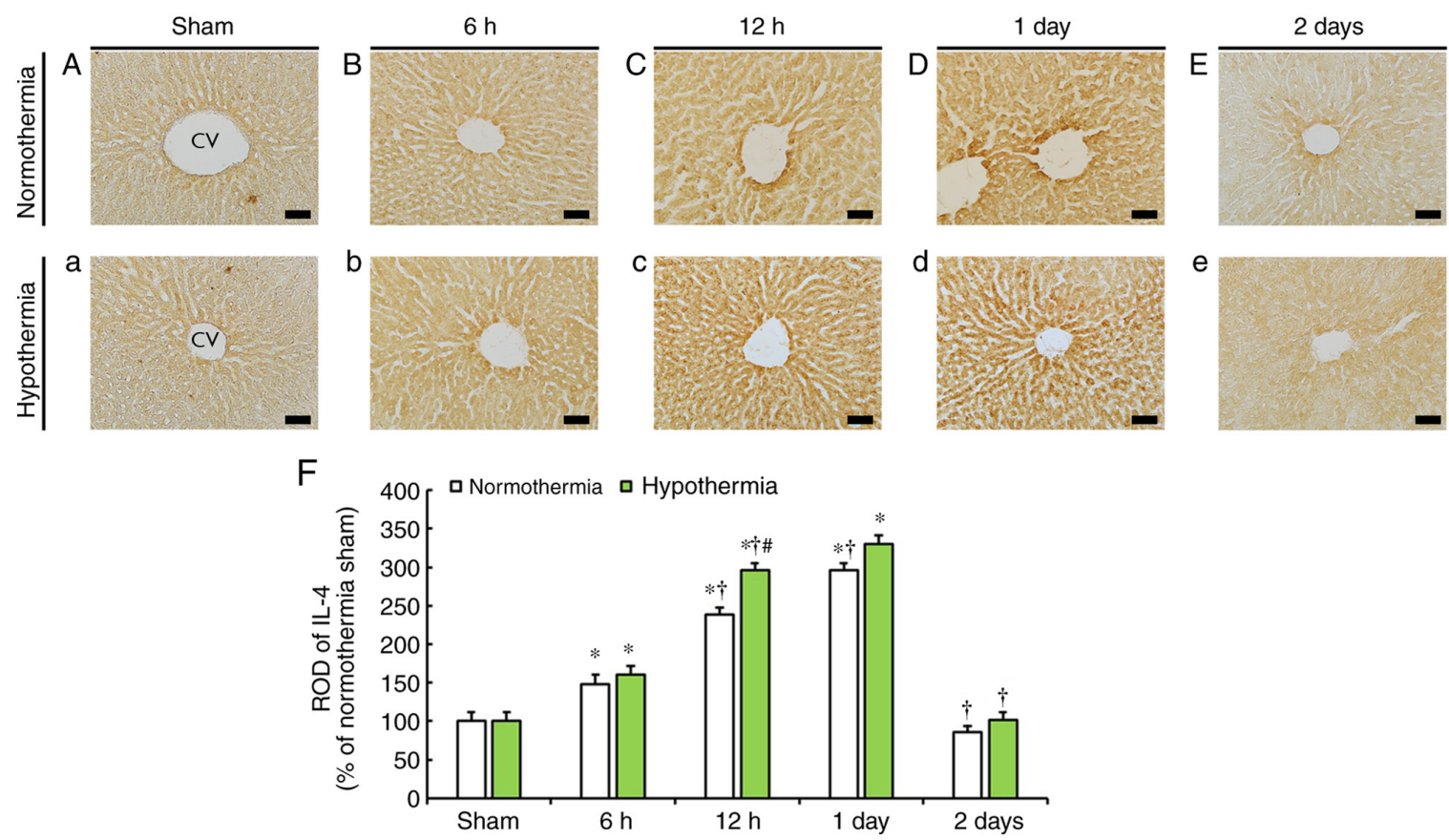

Figure 4. IL-4 immunoreactivity in the liver of the normothermia and hypothermia groups. (A-E) In the CA-operated normothermia and hypothermia groups, IL-4 immunoreactivity was gradually increased from $6 \mathrm{~h}$ post-CA, reached its highest level at 1 day post-CA and was notably reduced at 2 days post-CA, (a-e) while IL-4 immunoreactivity in the CA-operated hypothermia group was higher than that in the normothermia group. Scale bar, $50 \mu \mathrm{m}$. (F) ROD of IL-4 immunoreactivity in the normothermia and hypothermia groups. $\mathrm{n}=5$ (sham) or 7 (CA) per group. ${ }^{*} \mathrm{P}<0.05$ vs. the sham-operated normothermia or hypothermia group; ${ }^{\mathrm{P}} \mathrm{P}<0.05$ vs. the same group at the previous time point; ${ }^{*} \mathrm{P}<0.05$ vs. the normothermia group at the same time point. The data are presented as the mean \pm SEM. CV, central vein; ROD, relative optical density.
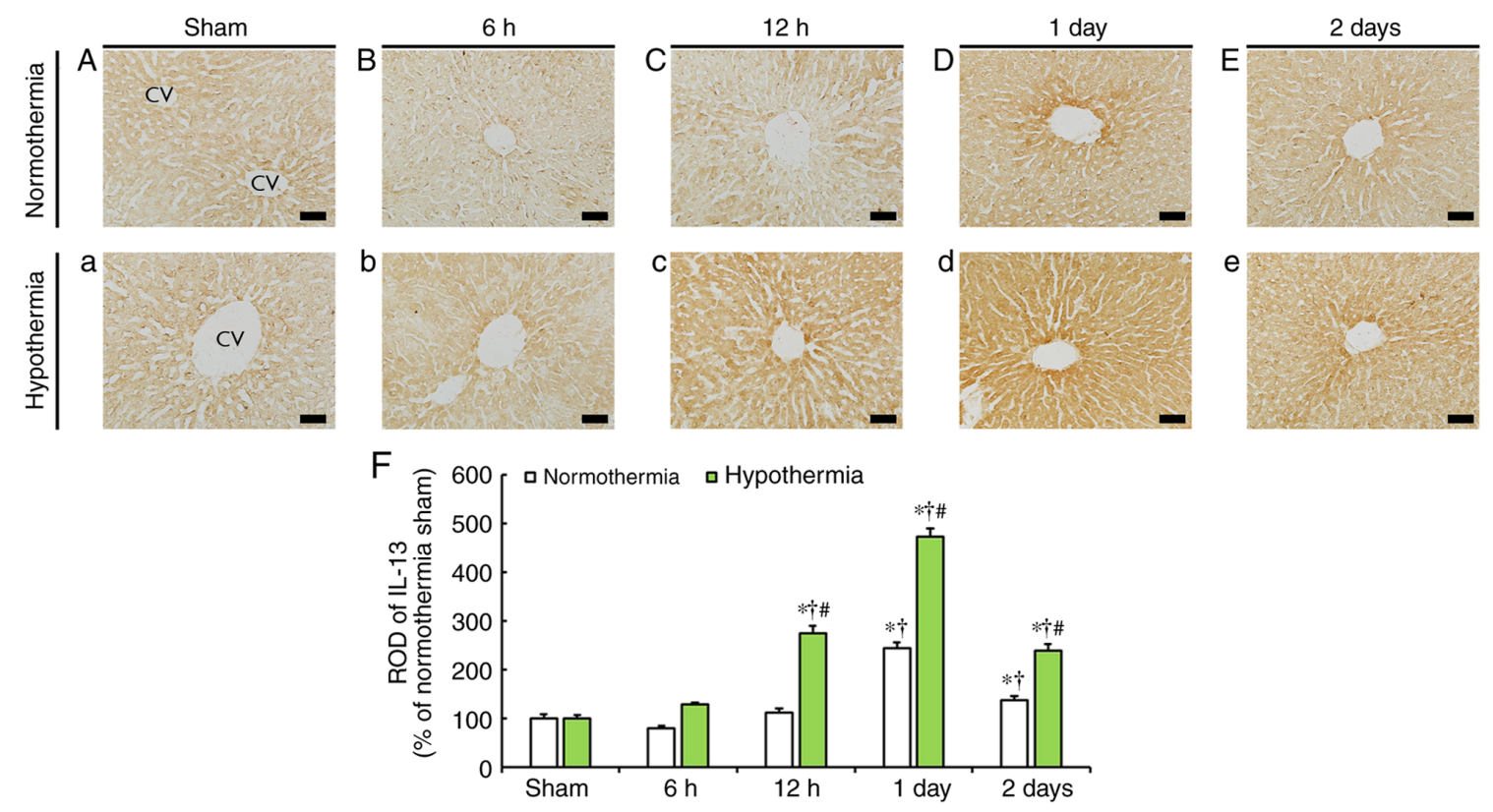

Figure 5. Immunoreactivity of IL-13 in the liver tissue of the normothermia and hypothermia groups. (A-E) In the CA-operated normothermia group, IL-13 immunoreactivity was not altered until $12 \mathrm{~h}$ after CA, while it was significantly enhanced at 1 day after CA and decreased at 2 days after CA. (a-e) In the CA-operated hypothermia group, IL-13 immunoreactivity at each time point was higher compared with that in the CA-operated normothermia group. Scale bar, $50 \mu \mathrm{m}$. (F) ROD of IL-13 immunoreactivity in the normothermia and hypothermia groups. $\mathrm{n}=5$ (sham) or 7 (CA) per group. "P<0.05 vs. the sham-operated normothermia or hypothermia group; ${ }^{\dagger} \mathrm{P}<0.05$ vs. the same group at the previous time point; ${ }^{\prime} \mathrm{P}<0.05$ vs. the normothermia group at the same time point. The data are presented as the mean \pm SEM. CV, central vein; ROD, relative optical density.

increased $(274.6 \%$ of the sham-operated hypothermia group) at $12 \mathrm{~h}$ post-CA and reached the highest level (472.3\% of the sham-operated hypothermia group) at 1 day post-CA (Fig. 5b, c, d and F). At 2 days post-CA, IL-13 immunoreactivity was decreased compared with the previous time point (Fig. 5e), but ROD was $238.7 \%$ of that in the sham-operated hypothermia group (Fig. 5F). 


\section{Discussion}

In the present study, the histopathological alterations and expression levels of pro- and anti-inflammatory cytokines in the liver following $5 \mathrm{~min}$ of asphyxial CA were examined. Hypothermic treatment for $4 \mathrm{~h}$ after $\mathrm{CA}$ was indicated to attenuate the histopathological damage, reduce the expression levels of pro-inflammatory cytokines and enhance the expression levels of anti-inflammatory cytokines compared with normothermic treatment.

In CA-induced normothermic livers, histopathological damage, including sinusoidal dilatation and vacuolization, became more apparent with time after CA. However, hypothermic treatment for $4 \mathrm{~h}$ after CA markedly attenuated these structural alterations in the liver. In accordance with the results of the current study, Behrends et al (16) have reported that hypothermic treatment protected against IR-induced liver injury. Taken together, these results indicated that the transient block of blood supply to the liver can evoke structural alterations in the liver. However, hypothermia after CA could decrease hepatocellular damage, therefore hypothermia exhibited protective effects against hepatic IR damage.

It has been reported that IL-2 administration in mice enhanced the activity of phagocyting Kupffer cells in the liver, causing leukocyte-endothelial adhesion and impeding sinusoidal microcirculation (31). These changes were indicated to induce hypoxic deterioration near central venules by inhibiting the hepatic sinusoidal blood flow (31). In addition, it has been reported that IL- 2 can induce secretion of TNF- $\alpha$ from hepatic Kuepfer cells, monocytes and macrophages $(31,32)$, suggesting that treatment with IL- 2 can biologically activate TNF- $\alpha$. TNF- $\alpha$ is a central pro-inflammatory cytokine produced from Kupffer cells in the liver or neutrophils recruited to the liver (33). It is known to be a primary inflammatory mediator in IR liver injury in rats (2). TNF- $\alpha$ has been indicated to induce the production of chemokines, ROS and adhesion molecules that are capable of causing direct cellular damage to the liver (8). TNF- $\alpha$ can also interact with TNF- $\alpha$ receptor 1 and activate signal transduction pathways of hepatocyte death (2). In the present study, 5 min of $\mathrm{CA}$ in the CA-operated normothermia group evoked a significant increase in TNF- $\alpha$ and IL-2 expression levels in the liver at 6 and $12 \mathrm{~h}$ after CA. At 1 and 2 days after CA, IL-2 expression was slightly decreased compared with the those at $12 \mathrm{~h}$ after $\mathrm{CA}$, whereas TNF- $\alpha$ expression was continuously increased in the liver, indicating that liver damage at 2 days post-CA under normothermia may be severe.

Hypothermic treatment for $4 \mathrm{~h}$ after CA significantly reduced the CA-induced increase in IL-2 and TNF- $\alpha$ expression levels, indicating a remarkable reduction in TNF- $\alpha$ expression at 1 and 2 days after CA compared with the normothermia group. These findings indicated that hypothermia resulted in the inhibition of the inflammatory response, which is typically observed following hepatic IR injury (8). It has been reported that inhibition of TNF- $\alpha$ production or its neutralization with a TNF- $\alpha$ antibody can decrease the number of neutrophils infiltrating into the liver after IR and rescue hepatic IR injury (33-35). In addition, it has been reported that administration of anti-TNF- $\alpha$ antibody improved the survival rate after treatment with a high dose of IL-2 in mice (31). Based on the results of the aforementioned studies and the current findings, it is likely that CA can induce an early increase in IL-2 and TNF- $\alpha$ expression levels in the liver under normothermia, while hypothermic treatment may be sufficient to reduce the IL-2 and TNF- $\alpha$ expression levels at later time points.

The present study revealed that IL-4 immunoreactivity in the CA-operated normothermia group was significantly increased from $6 \mathrm{~h}$ post-CA. It reached its highest level at 1 day post-CA and was markedly reduced at 2 days post-CA. IL-4 immunoreactivity in the CA-operated hypothermia group was altered in a similar pattern as in the normothermia group. However, the ROD level was significantly higher in the CA-operated hypothermia group than that in the normothermia group. In addition, IL-13 immunoreactivity in both groups was altered similarly to IL-4 immunoreactivity, with the ROD level in the hypothermia group being higher than that in the normothermia group. This finding suggested that anti-inflammatory cytokines may reduce the expression levels of pro-inflammatory cytokines. A previous study has demonstrated that IL-4 reduced the expression levels of pro-inflammatory cytokines, including TNF- $\alpha$ and IL-1, in hepatocytes following IR and it protected liver tissues against hepatic IR injury (36). In addition, IL-13 has been indicated to reduce liver damage following hepatic IR injury and lead to liver regeneration (37), and it was suggested that this protective role of IL-13 was mediated by the downregulation of the pro-inflammatory cytokine IL-2. Furthermore, it has been reported that the protective effect against hepatic IR injury was associated with STAT6 activation by IL-4 and IL-13, thereby suppressing NF- $\kappa \mathrm{B}$-dependent pro-inflammatory mediators $(9,38)$. Taken together, the current results indicated that IL- 4 may reduce TNF- $\alpha$ expression from $6 \mathrm{~h}$ post-CA and IL-13 may decrease IL-2 at later time points after $\mathrm{CA}$. In addition, the results of the present study suggested that hypothermia significantly increased the anti-inflammatory effect following IR injury compared with normothermia.

In summary, the present study revealed that CA significantly increased the expression levels of TNF- $\alpha$ and IL-2 from early time points post-CA and induced ischemic damage in the liver despite the increase in the expression levels of IL-4 and IL-13 in the ischemic liver under normothermia. However, hypothermic treatment after CA significantly increased IL-4 and IL-13 expression levels in the liver, which may reduce the expression levels of TNF- $\alpha$ and IL-2 and ameliorate CA-induced liver damage. These results indicated that hypothermic treatment after CA resulted in reduced hepatocellular damage, which was closely associated with decreased expression levels of pro-inflammatory cytokines and increased expression levels of anti-inflammatory cytokines in the liver after CA.

\section{Acknowledgements}

Not applicable.

\section{Funding}

The present study was supported by the National Research Foundation of Korea funded by the Korean government 
(Ministry of Science, ICT and Future Planning; grant no. 2017R1C1B5075773) and by Basic Science Research Program through the National Research Foundation of Korea funded by the Ministry of Education, Science and Technology (grant no. 2012R1A1A2001404).

\section{Availability of data and materials}

The datasets used and/or analyzed during the current study are available from the corresponding author on reasonable request.

\section{Authors' contributions}

YP, JHA, JunHC and MHW were responsible for the experimental design, data acquisition, data analysis and manuscript writing. JeongHC, HJT, TKL and BK performed the experiments and data analysis. JCL, JHP, MCS and TGO performed data analysis and made critical comments on the entire process of the study. All authors have read and approved the final version of manuscript. JunHC and MHW confirm the authenticity of all the raw data.

\section{Ethics approval and consent to participate}

All experimental protocols used in the present study were approved on the basis of ethical procedures and scientific care proposed by the Institutional Animal Care and Use Committee of Kangwon National University (approval no. KW-180124-1; January 2018; Chuncheon, South Korea).

\section{Patient consent for publication}

Not applicable.

\section{Competing interests}

The authors declare that they have no competing interests.

\section{References}

1. Kato A, Okaya T and Lentsch AB: Endogenous IL-13 protects hepatocytes and vascular endothelial cells during ischemia/reperfusion injury. Hepatology 37: 304-312, 2003.

2. Shuh M, Bohorquez H, Loss GE Jr and Cohen AJ: Tumor necrosis factor- $\alpha$ : Life and death of hepatocytes during liver ischemia/reperfusion injury. Ochsner J 13: 119-130, 2013.

3. Carr BG, Kahn JM, Merchant RM, Kramer AA and Neumar RW: Inter-hospital variability in post-cardiac arrest mortality. Resuscitation 80: 30-34, 2009.

4. Kida K, Shirozu K, Yu B, Mandeville JB, Bloch KD and Ichinose F: Beneficial effects of nitric oxide on outcomes after cardiac arrest and cardiopulmonary resuscitation in hypothermia-treated mice. Anesthesiology 120: 880-889, 2014.

5. Miao YF, Wu H, Yang SF, Dai J, Qiu YM, Tao ZY and Zhang XH: 5 -adenosine monophosphate-induced hypothermia attenuates brain ischemia/reperfusion injury in a rat model by inhibiting the inflammatory response. Mediators Inflamm 2015: 520745, 2015.

6. Niemann CU, Feiner J, Swain S, Bunting S, Friedman M, Crutchfield M, Broglio K, Hirose R, Roberts JP and Malinoski D: Therapeutic hypothermia in deceased organ donors and kidney-graft function. N Engl J Med 373: 405-414, 2015.

7. Niemann CU, Xu F, Choi S, Behrends M, Park Y, Hirose R and Maher JJ: Short passive cooling protects rats during hepatectomy by inducing heat shock proteins and limiting the induction of pro-inflammatory cytokines. J Surg Res 158: 43-52, 2010.
8. Abu-Amara M, Yang SY, Tapuria N, Fuller B, Davidson B and Seifalian A: Liver ischemia/reperfusion injury: Processes in inflammatory networks-a review. Liver Transpl 16: 1016-1032, 2010.

9. Eltzschig HK and Eckle T: Ischemia and reperfusion-from mechanism to translation. Nat Med 17: 1391-1401, 2011.

10. Lentsch AB, Kato A, Yoshidome H, McMasters KM and Edwards MJ: Inflammatory mechanisms and therapeutic strategies for warm hepatic ischemia/reperfusion injury. Hepatology 32: 169-173, 2000.

11. Jaeschke H, Bautista AP, Spolarics Z and Spitzer JJ: Superoxide generation by Kupffer cells and priming of neutrophils during reperfusion after hepatic ischemia. Free Radic Res Commun 15: 277-284, 1991.

12. Jaeschke H, Farhood A and Smith C: Neutrophils contribute to ischemia/reperfusion injury in rat liver in vivo. FASEB J 4: 3355-3359, 1990.

13. Black PR, van Devanter S and Cohn LH: Effects of hypothermia on systemic and organ system metabolism and function. J Surg Res 20: 49-63, 1976.

14. Xiao Q, Ye Q, Wang W, Xiao J, Fu B, Xia Z, Zhang X, Liu Z and Zeng $X$ : Mild hypothermia pretreatment protects against liver ischemia reperfusion injury via the PI3K/AKT/FOXO3a pathway. Mol Med Rep 16: 7520-7526, 2017.

15. Abdo EE, Figueira ER, Rocha-Filho JA, Chaib E, D'Albuquerque LA and Bacchella T: Preliminary results of topical hepatic hypothermia in a model of liver ischemia/reperfusion injury in rats. Arq Gastroenterol 54: 246-249, 2017.

16. Behrends M, Hirose R, Serkova NJ, Coatney JL, Bedolli M, Yardi J, Park YH and Niemann CU: Mild hypothermia reduces the inflammatory response and hepatic ischemia/reperfusion injury in rats. Liver Int 26: 734-741, 2006.

17. Eum HA, Cha YN and Lee SM: Necrosis and apoptosis: Sequence of liver damage following reperfusion after $60 \mathrm{~min}$ ischemia in rats. Biochem Biophys Res Commun 358: 500-505, 2007.

18. Lee JC, Tae HJ, Cho JH, Kim IS, Lee TK, Park CW, Park YE, Ahn JH, Park JH, Yan BC, et al: Therapeutic hypothermia attenuates paraplegia and neuronal damage in the lumbar spinal cord in a rat model of asphyxial cardiac arrest. J Therm Biol 83: $1-7,2019$.

19. Ahn JH, Lee TK, Kim B, Lee JC, Tae HJ, Cho JH, Park Y, Shin MC, Ohk TG, Park CW, et al: Therapeutic hypothermia improves hind limb motor outcome and attenuates oxidative stress and neuronal damage in the lumbar spinal cord following cardiac arrest. Antioxidants (Basel) 9: 38, 2020.

20. Kim YS, Cho JH, Shin MC, Park Y, Park CW, Tae HJ, Cho JH, Kim IS, Lee TK, Park YE, et al: Effects of regional body temperature variation during asphyxial cardiac arrest on mortality and brain damage in a rat model. J Therm Biol 87: 102466, 2020.

21. Cho JH, Tae HJ, Kim IS, Song M, Kim H, Lee TK, Kim YM, Ryoo S, Kim DW, Lee CH, et al: Melatonin alleviates asphyxial cardiac arrest-induced cerebellar Purkinje cell death by attenuation of oxidative stress. Exp Neurol 320: 112983, 2019.

22. National Research Council (US): Committee for the Update of the Guide for the Care and Use of Laboratory Animals: Guide for the Care and Use of Laboratory Animals. 8th edition. National Academies Press, Washington, DC, 2010.

23. Leary S, Underwood W, Anthony R, Corey D, Grandin T, Greenacre C, Gwaltney-Brant S, McCrackin MA, Meyer R, Miller D, et al: AVMA guidelines for the euthanasia of animals: 2013 edition. Journal, pp1-102, 2013.

24. Han F, Boller M, Guo W, Merchant RM, Lampe JW, Smith TM and Becker LB: A rodent model of emergency cardiopulmonary bypass resuscitation with different temperatures after asphyxial cardiac arrest. Resuscitation 81: 93-99, 2010.

25. Drabek T, Foley LM, Janata A, Stezoski J, Hitchens TK, Manole MD and Kochanek PM: Global and regional differences in cerebral blood flow after asphyxial versus ventricular fibrillation cardiac arrest in rats using ASL-MRI. Resuscitation 85: 964-971, 2014.

26. Hu T, Wang J, Wang S, Li J, Chen B, Zuo F, Zhang L, Huang Y and Li Y: Effects of the duration of postresuscitation hyperoxic ventilation on neurological outcome and survival in an asphyxial cardiac arrest rat model. Sci Rep 9: 16500, 2019.

27. Jia X, Koenig MA, Shin HC, Zhen G, Pardo CA, Hanley DF, Thakor NV and Geocadin RG: Improving neurological outcomes post-cardiac arrest in a rat model: Immediate hypothermia and quantitative EEG monitoring. Resuscitation 76 : 431-442, 2008. 
28. Lee CH, Park JH, Cho JH, Kim IH, Ahn JH, Lee JC, Chen BH, Shin BN, Tae HJ, Bae EJ, et al: Effect of oenanthe javanica extract on antioxidant enzyme in the rat liver. Chin Med J (Engl) 128 : $1649-1654,2015$

29. Carpenter JW: Exotic Animal Formulary. 4th edition. Elsevier Health Sciences, p744, 2012.

30. Park JH, Park O, Cho JH, Chen BH, Kim IH, Ahn JH, Lee JC, Yan BC, Yoo KY, Lee CH, et al: Anti-inflammatory effect of tanshinone I in neuroprotection against cerebral ischemia-reperfusion injury in the gerbil hippocampus. Neurochem Res 39: $1300-1312,2014$.

31. Nakagawa K, Miller FN, Sims DE, Lentsch AB, Miyazaki M and Edwards MJ: Mechanisms of interleukin-2-induced hepatic toxicity. Cancer Res 56: 507-510, 1996.

32. Strieter RM, Remick DG, Lynch JP III, Spengler RN and Kunkel SL: Interleukin-2-induced tumor necrosis factor-alpha (TNF-alpha) gene expression in human alveolar macrophages and blood monocytes. Am Rev Respir Dis 139: 335-342, 1989.

33. Li J, Ke W, Zhou Q, Wu Y, Luo H, Zhou H, Yang B, Guo Y, Zheng $\mathrm{Q}$ and Zhang Y: Tumour necrosis factor- $\alpha$ promotes liver ischaemia-reperfusion injury through the PGC-1 $\alpha / \mathrm{Mfn} 2$ pathway. J Cell Mol Med 18: 1863-1873, 2014.
34. Colletti LM, Remick DG, Burtch GD, Kunkel SL, Strieter RM and Campbell DA Jr: Role of tumor necrosis factor-alpha in the pathophysiologic alterations after hepatic ischemia/reperfusion injury in the rat. J Clin Invest 85: 1936-1943, 1990.

35. Yang YL, Li JP, Xu XP, Dou KF, Yue SQ and Li KZ: Protective effects of tumor necrosis factor alpha antibody and ulinastatin on liver ischemic reperfusion in rats. World J Gastroenterol 10: 3161-3164, 2004

36. Kato A, Yoshidome H, Edwards MJ and Lentsch AB: Reduced hepatic ischemia/reperfusion injury by IL-4: Potential anti-inflammatory role of STAT6. Inflamm Res 49: 275-279, 2000.

37. Cannistrà $M$, Ruggiero $M$, Zullo A, Gallelli G, Serafini $S$, Maria M, Naso A, Grande R, Serra R and Nardo B: Hepatic ischemia reperfusion injury: A systematic review of literature and the role of current drugs and biomarkers. Int J Surg 33 (Suppl 1): S57-S70, 2016.

38. Girotra S, Chan PS and Bradley SM: Post-resuscitation care following out-of-hospital and in-hospital cardiac arrest. Heart 101: 1943-1949, 2015.

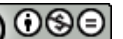

This work is licensed under a Creative Commons Attribution-NonCommercial-NoDerivatives 4.0 International (CC BY-NC-ND 4.0) License. 